\title{
STEM- and EFTEM-Analysis of Nanomaterials at High Spatial and High Energy Resolution
}

\author{
W. Grogger, B. Schaffer, M. Rogers, G. Kothleitner, and F. Hofer
}

Research Institute for Electron Microscopy, Graz University of Technology, Steyrergasse 17, A8010 Graz, Austria

The full characterization of nanostructured devices and functional nanomaterials at a nanometer scale is paramount for the understanding of their electrical, optical, or mechanical properties. Transmission electron microscopy (TEM) offers an excellent tool for this job, in terms of structural and chemical analysis on a nearly atomic scale. In particular, electron energy loss spectrometry (EELS) can obtain information not only about the chemical composition of a sample, but also about chemical bonding (via energy loss fine structure, ELNES) and optical properties (through valence loss EELS, VEELS) [1-4]. Recent instrumental improvements like TEM monochromators have made it possible to considerably improve energy resolution for EELS, which makes the spectral quality comparable to that of X-ray absorption spectrometry and allows for the detection of subtle ELNES changes [5]. Another strong point of the method lies in the combination with a fine electron probe (STEM), where high spatial resolution can be combined with high energy resolution EELS. A special advantage of this combination can be the simultaneous gain of spectral and spatial information, which can be done spectrum per spectrum (STEM-EELS spectrum imaging) or image per image (EFTEM spectrum imaging).

All results presented in this paper were obtained on a monochromated $200 \mathrm{kV}$ FEG TEM/STEM (FEI) with a high resolution imaging filter attached to it (HR-GIF, Gatan) [6, 7]. The obtainable energy resolution of this instrument is $0.2 \mathrm{eV}$. We have done experiments using both approaches in order to fill the three-dimensional data cube. For EFTEM spectrum imaging we have developed acquisition and correction algorithms that are necessary for measuring a reliable data set as well as for interpreting the data correctly and accurately. In this mode with the monochromator switched off we were able to get spatial resolution values below $1 \mathrm{~nm}$ and an energy resolution of $0.8 \mathrm{eV}$ due to the precise slit assembly of the HR-GIF. In addition we also used STEM spectrum imaging in combination with the monochromator. In this mode spatial resolution was limited by the properties of the monochromated illumination system of the TEM to values of about $2 \mathrm{~nm}$. At the same time EEL spectra could be acquired with less than $0.25 \mathrm{eV}$.

As a real world application example we investigated a $\mathrm{VN}_{\mathrm{x}}$ coating on a Si substrate in STEM mode (fig. 1a) [8,9]. The energy resolution in this particular case was $0.27 \mathrm{eV}$ measured as the FWHM of the zero loss peak. By looking at the ELNES of the N K ionization edge we could clearly differentiate between $\mathrm{VN}$ and $\mathrm{V}_{2} \mathrm{~N}$ (fig. 1b) and compare these results with theoretical simulations. Fig. 2a shows a bright field image of an InAs/In(P,As) multilayer structure. The nominal thicknesses are $4 \mathrm{ML}$ for the InAs and $20 \mathrm{~nm}$ for the $\mathrm{In}(\mathrm{P}, \mathrm{As})$. There is a slight plasmon shift of about $0.2 \mathrm{eV}$ between the two compounds. Using an EFTEM series with an extremely small slit width of $0.4 \mathrm{eV}$ in the energy range up to $25 \mathrm{eV}$ this region could be evaluated in terms of the position of the plasmon with superb energy precision (fig. 2b).

The presentation of the paper will focus on the aspects important for spatial and energy resolution. We will point out the limitations of current commercially available TEM instruments and demonstrate the use of the methods with some practical application examples [10]. 


\section{References}

[1] F. Hofer, P. Warbichler, in Transmission Electron Energy-Loss Spectroscopy in Materials Science, Plenum Press, New York, 2004.

[2] W. Grogger et al., Ultramicroscopy 96 (2003) 469.

[3] R. Brydson, Mat. Sci Technol. 16 (2000) 1187

[4] R.H. French, J. Am. Ceram. Soc. 73 (1990) 477.

[5] C. Mitterbauer et al., Ultramicroscopy 96 (2003) 469.

[6] P. Tiemeijer et al., Microsc.Microanal. 7 (Suppl. 2: Proc.) (2001) 1130.

[7] H.A. Brink et al., Ultramicroscopy 96 (2003) 367.

[8] G. Kothleitner et al., Appl. Surf. Sci. (2005) in print

[9] G. Kothleitner et al., Prakt. Met. (2005) in print

[10] Holm Kirmse, Humbolt University Berlin, is gratefully acknowledged for providing the InAs/In(P,As) sample. This work was financially supported by the SFB "Electroactive Materials", Austrian Science Fund Vienna.
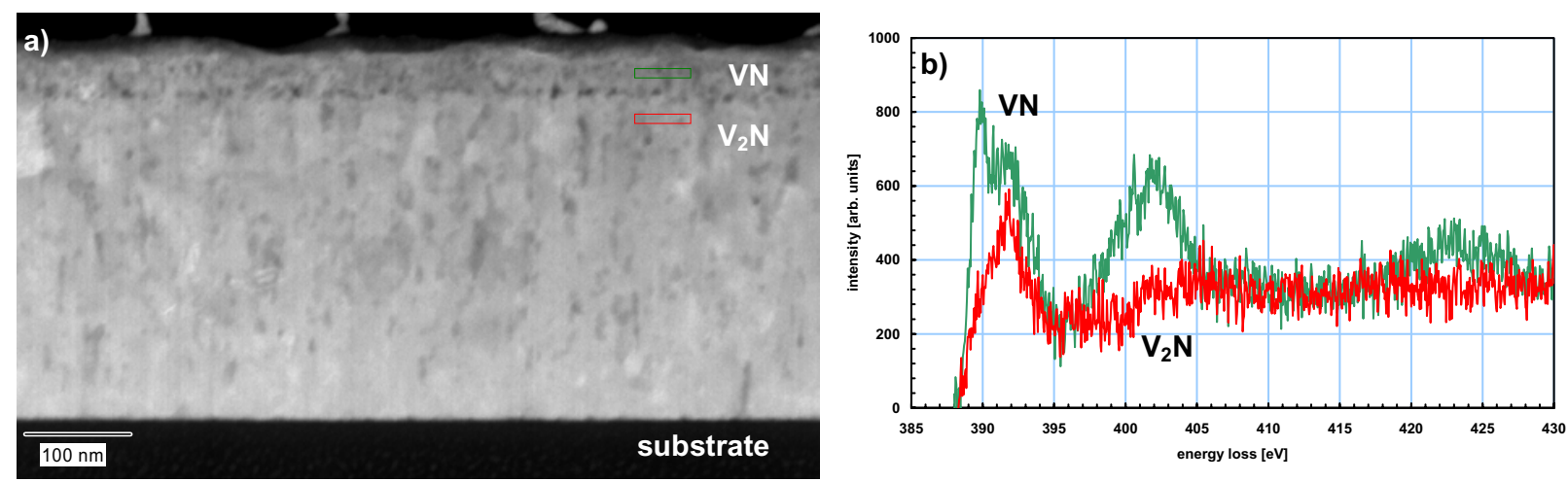

Figure 1: a) HAADF STEM image of $\mathrm{VN}_{\mathrm{x}}$ coatings on silicon; b) ELNES of the $\mathrm{N} \mathrm{K}$ ionization edge for $\mathrm{VN}$ and $\mathrm{V}_{2} \mathrm{~N}$ acquired with $0.27 \mathrm{eV}$ energy resolution.
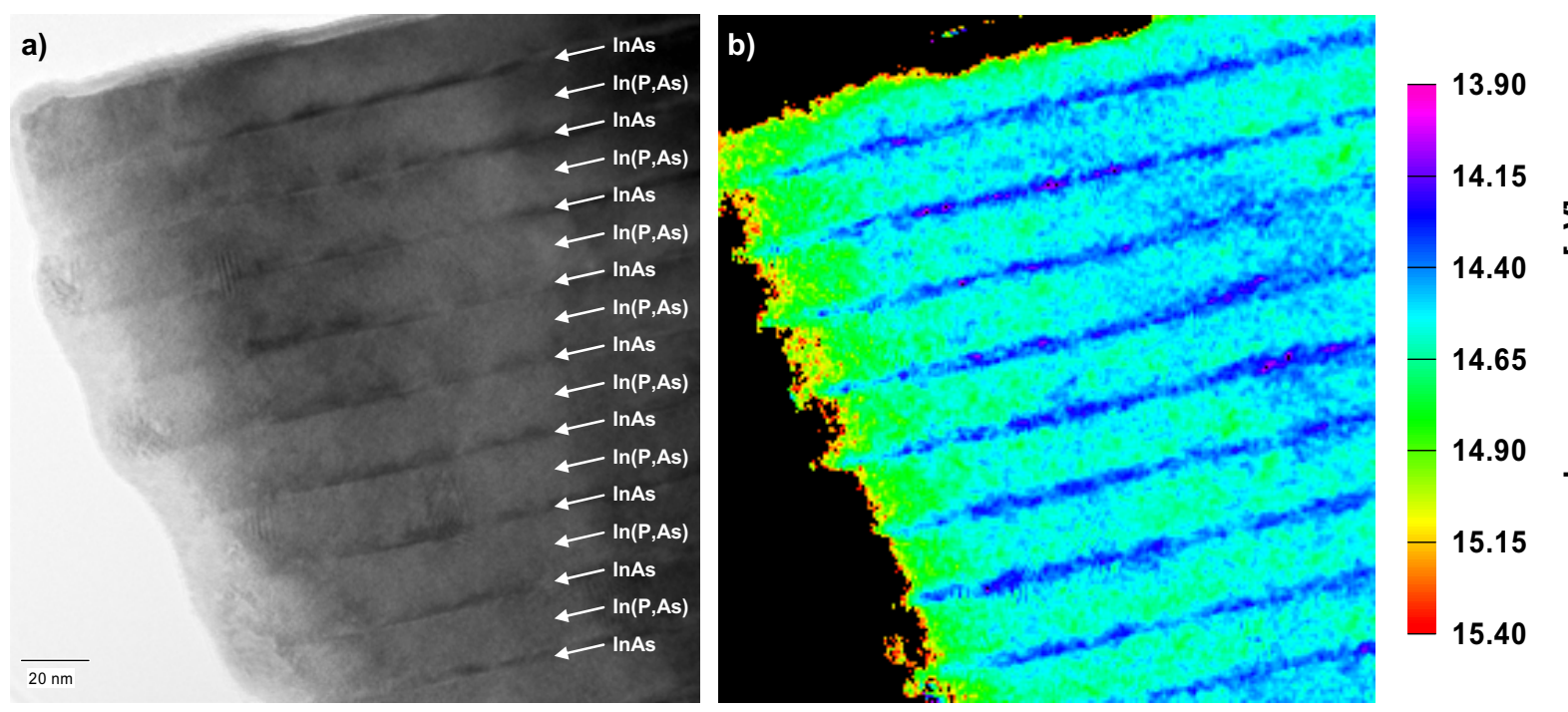

Figure 2: a) TEM bright field image of a $\operatorname{InAs} / \operatorname{In}(\mathrm{P}, \mathrm{As})$ multilayer; b) plasmon energy map calculated from a high energy resolution EFTEM series. The energy difference of the plasmons of InAs and $\operatorname{In}(\mathrm{P}, \mathrm{As})$ is only $0.2 \mathrm{eV}$. 